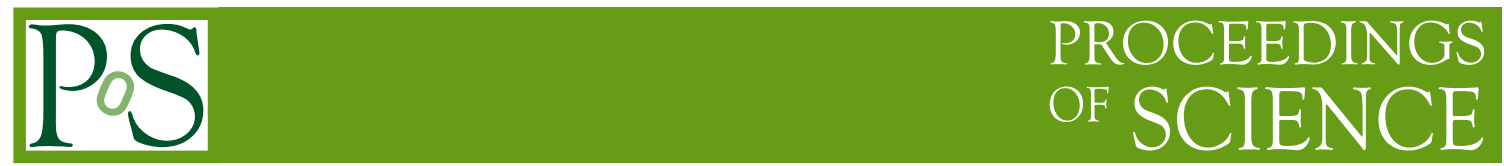

\title{
Galactic and Extragalactic Novae - An Updated Review
}

\section{Rosa Poggiani*}

Università di Pisa and Istituto Nazionale di Fisica Nucleare, Sezione di Pisa

E-mail: rosa.poggiani@df .unipi.it

The eruptions of novae are among the brightest explosions on the Universe. I present a review of the recent observations of galactic and extragalactic novae, with a focus on the results of the new facilities for high cadence optical observations and of X-ray and gamma ray observatories.

The Golden Age of Cataclysmic Variables and Related Objects IV 11-16 September, 2017

Palermo, Italy

${ }^{*}$ Speaker. 


\section{Introduction}

Classical novae are cataclysmic variable systems harboring a white dwarf accreting material from a secondary star [85], [15], [10]. Novae undergo outbursts whose typical signature is a rapid increase of the optical luminosity. The nova outbursts are produced by a ThermoNuclear Runaway (TNR) on the surface of the white dwarf [15], [10], [133], [39]. The historical accounts of nova eruptions mainly report optical observations, that are presently performed with high cadence photometry and spectroscopy. The emission of novae is not limited to the optical domain, but spans the whole electromagnetic spectrum, making them suitable sources for multifrequency investigation with ground based and satellite based instruments [41]. The X-ray and gamma ray observations have revealed a rich pattern of physical processes. Novae have also been considered as candidate standard candles. A general introduction to the topics related to cataclysmic variables and novae can be found in [41].

In this paper, I review some aspects of multifrequency observations of Galactic and extragalactic novae, with an emphasis on the recent facilities for high cadence optical observations and high energy instruments in the X-ray and gamma ray domains.

\section{Novae: summary of basic properties}

I will firstly summarize the main features of novae in the optical domain, since they are the basis for their classification. Galactic novae have been cataloged by [37], [36], [101], [102]. A living catalog of the recent novae is being maintained by [71]. To date, more than four hundreds Galactic novae are known. The majority of extragalactic novae has been observed in the close galaxies. The catalogs of extragalactic novae [86], updated at July 2018, contain 1118 novae in $\mathrm{M} 31^{1}, 51$ in $\mathrm{M}_{3}{ }^{2}, 53$ in $\mathrm{LMC}^{3}, 22$ in $\mathrm{SMC}^{4}$.

The light curve of novae during the decline is not generally smooth, but can show dips, flares etc. The photometric evolution of novae has been addressed by [134], who proposed a classification system into seven prototype classes (Fig. 1): the F-class novae with flat top ( $2 \%$ of all novae); the C-class novae with a cusp (1\%); the J-class novae with jitters $(16 \%)$; the P-class novae with a plateau (21\%); the O-class novae with oscillations (4\%); the S-class system with a featureless decline (38\%); the D-class novae with dips (18\%). The model by [44], [57] suggests a universal decline law for novae that do not show peculiar features during the early stages. The universal light curve behaves as $\mathrm{t}^{-1.75}$ in the time interval corresponding to the drop from to 2 to 6 magnitudes below the maximum and as $\mathrm{t}^{-3.5}$ in the interval corresponding to the drop from 6 to 10 magnitudes below maximum, where $t$ is the time elapsed from the eruption in days.

Photometric observation for most novae are available at the archives of AAVSO ${ }^{5}$ and VSNET ${ }^{6}$. Recently, all-sky surveys monitoring the variability of a large number of known and new transient

\footnotetext{
${ }^{1}$ http://www.mpe.mpg.de/ m31novae/opt/m31/index.php

${ }^{2}$ http://www.mpe.mpg.de/ m31novae/opt/m33/index.php

${ }^{3}$ http://www.mpe.mpg.de/ m31novae/opt/lmc/index.php

${ }^{4}$ http://www.mpe.mpg.de/ m31novae/opt/smc/index.php

${ }^{5}$ https://www.aavso.org/

${ }^{6}$ http://ooruri.kusastro.kyoto-u.ac.jp/mailman/listinfo/
} 


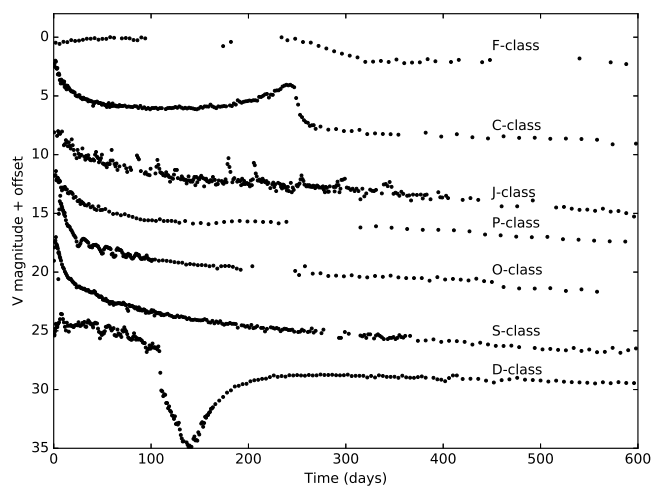

Figure 1: Classification of the light curves of novae, after [134]; data from [134]

sources with high temporal cadence have started operations. The determination of the orbital period of novae and the investigation of the pre-maximum stage are among the science topics allowed by a dense time sampling. The space based Solar Mass Ejection Imager (SMEI) instrument secures photometric observations with a cadence of about 100 minutes. The imager has measured the light curves of 13 galactic novae [50], [51], including some objects detected by ground based instruments only after the outburst. Some novae have been observed during the poorly studied pre-maximum stage (see [99] for a review of the pre-maximum stage). The ground based Optical Gravitational Lensing Experiment (OGLE) has monitored 39 Galactic novae in the Galaxy [68] (about one third of the sample has been discovered by the survey), detected 80 post-nova candidate systems and estimated the orbital periods of 18 objects.

The classification of novae is based on observations in the optical domain. The original spectral classification by [146], [147], [148] is based on the presence of specific lines (in addition to the Balmer lines) and suggests two separate classes, the Fe II novae and the He/N that show Fe II multiplets or $\mathrm{He}$ and $\mathrm{N}$ lines, respectively, in the early post-outburst spectra. The spectral differences are mirrored by different photometric properties and different physical mechanisms. Fe II novae include about $80 \%$ of Galactic novae and show slow to moderately fast declines, evolving from early spectra with strong Fe II lines to late spectra with nebular lines; the underlying mechanism is wind ejection. He/N novae show fast declines and higher brightness, switching from early spectra with $\mathrm{He}$ and $\mathrm{N}$ lines to nebular spectra with coronal lines; the physical mechanism is shell ejection. An example of the post-outburst spectra of Fe II and He/N novae is shown in Fig. 2. In addition to showing different spectra, the Fe II and He/N novae show a different photometric evolution: the former are fainter and slower than the latter [34]. Galactic novae belonging to the two classes show also a different spatial distribution: He/N novae concentrate close to the Galactic plane [28], [34].

Some novae have been classified in the hybrid class, since their spectra evolve from the Fe II class to He/N class. Recently, V5558 Sgr [136] and T Pyx (see e.g. [54], [40]) showed the opposite behavior, evolving from a He/N spectrum to a Fe II spectrum. According to [151], the origin of the $\mathrm{He} / \mathrm{N}$ and $\mathrm{Fe}$ II spectra is in the white dwarf ejecta or in a circumbinary gas envelope around the secondary star. The observation of the two types of spectra in the same object can be explained with a time varying contribution of the above mechanisms. Most novae should exhibit both type of spectra during their evolution [151]. 

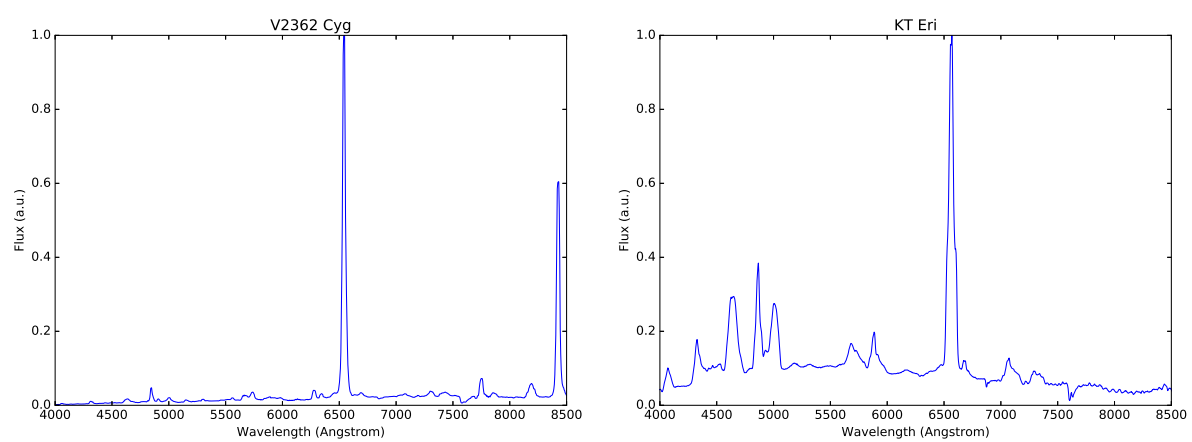

Figure 2: Spectra of the Fe II nova V2362 Cyg [90] He/N nova KT Eri (Poggiani, in preparation) during the initial decline

The SMARTS Consortium [144], [145] has built a living spectral atlas of Southern novae, the successor of the historical Tololo nova atlas [148], [149]. In 2017 the atlas contained the optical/IR photometric and spectroscopic data of more than seventy novae, observed with the ANDICAM dual channel imager and the RC spectrograph at the SMARTS $1.5 \mathrm{~m}$ telescope [144]. The atlas has allowed some investigations [145]. Dust forming novae do not necessarily show a dip in the decline curve, while the $\mathrm{K}_{s}$ band flux increases before the appearance of the dip also in novae where dust formation is not associated to a brightness drop [145]. The simultaneous monitoring with SMARTS and Swift has shown that intensity of He II 4686 in He/N novae increases just before the onset of the super-soft phase in the X-rays and decreases at the turn-on [145].

Since 2005 I am monitoring Northern novae at the Cassini 1.5m telescope, equipped with the BFOSC Imager/Spectrograph [96]. Optical spectra are secured during the decline with a high cadence. The sample includes more than twenty galactic novae and some extragalactic novae: V1663 Aq1 [87], V1722 Aq1, V809 Cep, V962 Cep, V2362 Cyg [90], V2467 Cyg [91], V2468 Cyg, V2491 Cyg, V2659 Cyg, V407 Cyg, V339 Del, KT Eri, V959 Mon, V2615 Oph, V2670 Oph [92], V2944 Oph, V496 Sct, V612 Sct (ASASSN-17hx) [99], V556 Ser, V5558 Sgr [88], [93], V5584 Sgr [95], [98], V458 Vul [89], V459 Vul [94], the extragalactic novae M31 2009-10b, M31 2010-07a, M31 2011-07 and M33 2010-07a [97]. Among the observed sample, a few objects showed a peculiar photometric or spectroscopic behavior. V458 Vul is an hybrid nova that evolved from the Fe II to the He/ $\mathrm{N}$ class after the outburst [89]. V2362 Cyg showed a secondary brightening during the decline, produced by a secondary mass ejection [90]. V2467 Cyg showed oscillations during the decline [91]. V5558 Sgr is a very slow nova harboring a white dwarf with a mass at the lower limit to trigger a nova outburst [88], [93]. V612 Sct (ASASSN-17hx) is a slow nova with a long duration pre-maximum [99]. M31 2009-10b is one of the brightest novae ever observed in M31 [97], while M33 2010-07a is the first nova in M33 that showed a secondary mass ejection [97].

Novae are classified into classical novae and recurrent novae, according to the number of observed eruptions [10]. The recurrence time of outbursts ranges from tens to hundred years in recurrent novae and is larger than thousand years for classical novae. The interval between outbursts is governed by the mass of the white dwarf and the accretion rate [152]. Recurrent novae show an high rate of mass transfer that explains the short recurrence times [78] and are possible progenitors 
of type Ia supernovae [135], [118]. There are 10 known recurrent Galactic novae [108], 4 in LMC [78] and 12 in M31 [118]. The photometric history of Galactic recurrent novae has been presented by [108]. An example is the light curve of CI Aq1 [108] shown in Fig. 3.

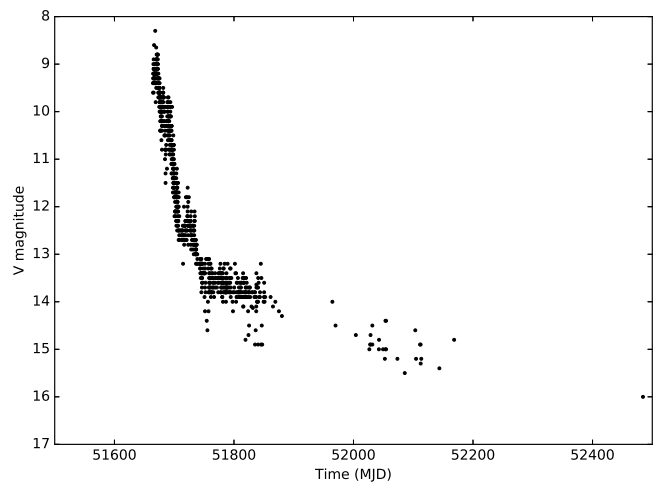

Figure 3: The historical light curve of CI Aql; data from [108]

Recurrent novae are classified into three classes. T Pyx, IM Nor and CI Aql have orbital periods smaller than one day and main sequence secondary stars. The systems U Sco, V394 CrA and V2487 Oph have orbital periods of the order of one day and evolved secondaries. The objects T CrB, RS Oph, V745 Sco and V3890 Sgr have orbital periods of the order of years and red giant secondaries. Most recurrent novae belong to the $\mathrm{He} / \mathrm{N}$ spectral class.

The fraction of recurrent novae in M31 is of the order of a few percent [118]. Among them, nova M31 2008-12a underwent ten recorded outbursts, thanks to its short recurrence time, that could be either $351 \pm 13$ days or one half of the value, $175 \pm 11$ days [48]. The white dwarf mass of the system is close to the Chandrasekhar limit, making M31 2008-12a a supernova Ia progenitor candidate. The outbursts have triggered multifrequency observations with ground and space based instruments. The optical, infrared and X-ray monitoring of the 2015 outburst has been reported by [25]. The predicted 2016 and 2017 outbursts have been observed [55], [11].

\section{Galactic and extragalactic nova rates}

The search for different extragalactic nova populations has been addressed by surveys in the close galaxies M31, M33, LMC, M87. The properties of extragalactic novae have been reviewed by [117]. In the following, an update of the nova rates in some close galaxies will be given.

The existence of two nova populations in M31, in disk and in bulge, has been proposed by [24]. A spectroscopic survey of the M31 bulge has been performed by [139], showing an agreement with the spectral evolution of the Galactic novae. The survey by [114] showed that novae belonging to the $\mathrm{Fe}$ II and to the He/ $\mathrm{N}$ class were $82 \%$ and $18 \%$, as in Galactic novae. A spectroscopic survey of novae in M33 has been performed by [115], who showed that 5 and 2 novae out of 8 were He/N and Fe II novae, respectively, showing a clear departure from the populations in the Galaxy and in M31. A survey of novae in LMC has been performed by [116], who observed a similar fraction of novae belonging to the Fe II and He/N class. The large fraction of He/N novae in M33 and LMC is 
explained by the young stellar population, that produces more massive white dwarfs and an higher number of recurrent novae.

An analysis of the Galactic nova rate has been presented by [119], who estimated a rate of $50_{-23}^{+31} \mathrm{yr}^{-1}$. Previous estimations of the rate are reported in Table 1.

\begin{tabular}{lr}
\hline Authors & Rate $\left(\mathrm{yr}^{-1}\right)$ \\
\hline Allen 1954 [5] & 100 \\
Sharov 1972 [129] & 260 \\
Liller and Mayer 1987 [60] & $73 \pm 24$ \\
Della Valle 1988 [27] & $15 \pm 5$ \\
Ciardullo et al. 1990 [18] & 11 to 46 \\
van den Bergh 1991 [142] & 16 \\
Della Valle and Livio 1994 [32] & 20 \\
Hatano et al. 1997 [46] & $41 \pm 20$ \\
Shafter 1997 [111] & $35 \pm 11$ \\
Shafter 2002 [113] & $36 \pm 13$ \\
Matteucci et al. 2003 [63] & 25 \\
Mroz et al. 2015 [68] & $13.8 \pm 2.6$ \\
Shafter 2017 [119] & $50_{-23}^{+31}$ \\
\hline
\end{tabular}

Table 1: Rates of Galactic novae reported in literature

The estimated nova rates in M31 are presented in Table 2. Due to saturation of photographic plates, the surveys by [52], [6], [104], [105], [106] missed novae in the region of the nucleus. Nova events can occur in any part of M31 [18], with the largest density in the halo and in the bulge [17], [13].

\begin{tabular}{lr}
\hline Authors & Rate $\left(\mathrm{yr}^{-1}\right)$ \\
\hline Hubble 1929 [52] & 30 \\
Arp 1956 [6] & 26 \\
Capaccioli et al. 1989 [13] & $29 \pm 4$ \\
Shafter and Irby 2001 [120] & $37_{-8}^{+12}$ \\
Darnley et al. 2006 [24] & $65_{-15}^{+16}$ \\
\hline
\end{tabular}

Table 2: Nova rates in M31

Since M33 is an early type galaxy, it should show an higher nova rate than M31 [29]. The estimated rate span an interval from less than $0.4 \mathrm{yr}^{-1}$ [130] to $4.7 \pm 1.5 \mathrm{yr}^{-1}$ [29]. The most recent estimation by [150] is $2.5_{-0.7}^{+1.0} \mathrm{yr}^{-1}$. The estimations of the nova rates in LMC are clustered around a few novae per year: 2 to 3 novae per year [42], $2 \pm 1 \mathrm{yr}^{-1}$ [14], $2.5 \pm 0.5 \mathrm{yr}^{-1}$ [30], 2.4 $\pm 0.8 \mathrm{yr}^{-1}$ [69]. The estimations of the rate in M87 are scattered: $91 \pm 34 \mathrm{yr}^{-1}$ [112]; $154_{-19}^{+23} \mathrm{yr}^{-1}$ [23]; $363_{-45}^{+33}$ $\mathrm{yr}^{-1}$ [124].

The estimated extragalactic nova rates are compared by normalizing them to the stellar mass surveyed [117] via the galaxy K-band luminosity, producing the Luminosity Specific Nova Rates (LSNR). The rates in galaxies belonging to different Hubble types has been discussed by [153], [63]. The nova rate is proportional to the K-band luminosity of the galaxy (Fig. 4). 


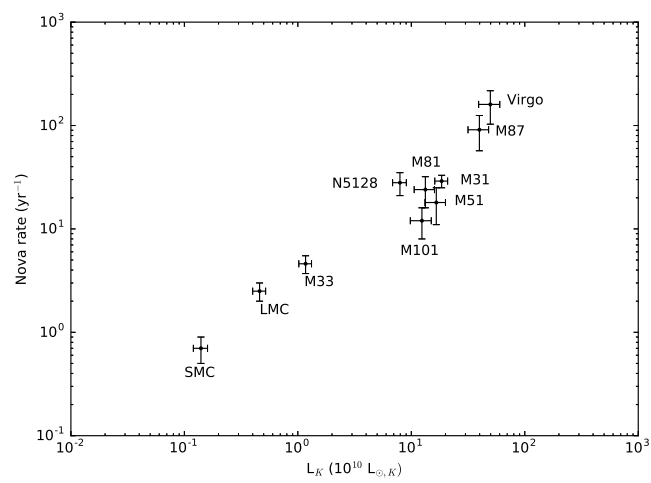

Figure 4: Relation between the nova rate in a galaxy and the K-band luminosity; data from [63]

\section{Novae and cataclysmic variables}

The orbital period of novae has been measured only for a small fraction of the observed systems. The distribution of measured periods of novae built using the data by [101], [102] is shown in Fig. $5^{7}$. The distribution has a peak in the region between 3 and 4 hours, above the period gap of cataclysmic variables. As discussed by [138], novae show an high mass accretion rate onto the white dwarf.

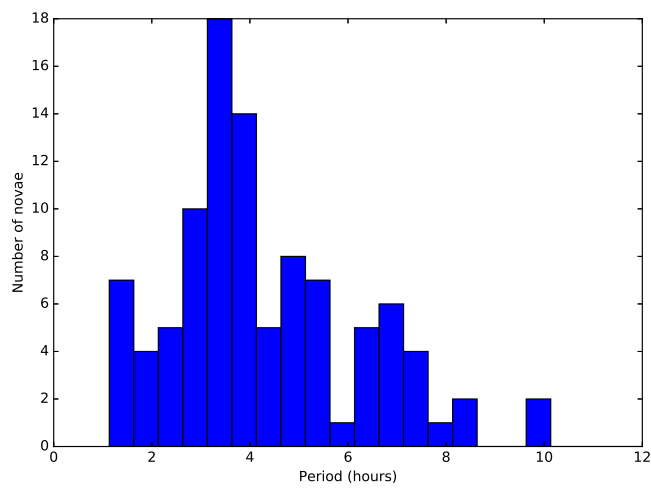

Figure 5: Distribution of the orbital periods of Galactic novae, data from [102]

The evolution of novae over long time scales has been discussed by [84]. The pre-outburst light curves has been studied by [103] and [22], who showed that the majority of novae shares the same quiescent magnitude before and after the eruption. According to [22], only a few novae showed a larger post eruption brightness.

The history of novae between eruptions has been debated for a long time. It has been proposed that novae could enter hibernation and move into a low mass transfer state [121] after the high mass loss outburst. The detachment of the secondary star from its Roche lobe is reversed during the hibernation stage, when the combination of gravitational wave emission and magnetic braking progressively reduces the orbital radius. The irradiation by the white dwarf produces a relatively

\footnotetext{
${ }^{7}$ Novae with evolved secondary stars and periods above 10 hours have been excluded
} 
high brightness for decades after the outburst. When the irradiation fades, the accretion rate decreases and dwarf nova outbursts appear [121]. The hibernation model predicts that novae undergo a cyclical evolution between states with high and low mass transfer rate [121], with hibernation intervals lasting for hundreds thousands years at least. The model predictions are in agreement with the observed decline rates of old novae [38]. An additional support to the model is given by the observation of shells around the dwarf novae Z Cam [122], AT Cnc [123]. In particular, the last nova eruption of AT Cnc occurred about three hundreds years ago [127]. The dwarf nova in Te 11 nebula has been associated to the nova $483 \mathrm{CE}$ in Orion, with the nebula being the remnant of the ourburst [66]. Recently, nova shells have been discovered around two nova-like variables, V1315 Aq1 [107] and IPHASX J210204.7+471015 [43]. Another support to the hibernation model is the observation of dwarf nova outbursts in systems labeled as novae. V1213 Cen has been observed during the pre-maximum and the outburst stages by [70], who detected the awakening of the object from the hibernation stage. After the 2009 outburst, V1213 Cen faded until 2016. The pre-eruption data showed some dwarf nova outbursts, with an amplitude of about three magnitudes. The quiescent brightness is compatible with the brightness of dwarf novae. The nova that erupted on 11 March AD 1437 showed dwarf nova outbursts after five centuries [125].

There is an ongoing effort to recover systems labeled as old novae in the catalogs of cataclysmic variables [36], sometimes missing the spectroscopic identification. The mining of the plate archives of world observatories, that contain records of astronomical data that can extend for decades, is being performed by [53], [83]. The observational search for old novae is based on multicolour photometry followed by spectroscopic observations [137], [83]. Old novae are selected by their position in the color-color diagram, far from the main sequence curve [137]. The spectroscopic follow-up allows the classification of the object as a nova or a generic cataclysmic variable.

\section{Distances}

Novae have been considered as candidate standard candles for the estimation of extragalactic distances [140], [33]. The Very Large Telescope (VLT) has been used to search for novae in NGC 1316, an early type galaxy in the Fornax cluster [31] at about $20 \mathrm{Mpc}$.

The method of the Maximum Magnitude Rate of Decline (MMRD) [21], [33], [26], [35], [109] has been recently rediscussed. A new class of faint and fast novae has been discovered in the high cadence survey of M 31, M 81, M 82, NGC 2403, NGC 891 by [56]. The luminosity and decay time of the detected novae were not consistent with the standard MMRD relation. While fast Galactic novae are brighter than slow novae and belong to the $\mathrm{He} / \mathrm{N}$ spectral class, the detected fast and faint novae were Fe II novae. Faint and fast novae are common also in M87, being about $40 \%$ of all novae and showing a great scatter around the MMRD relation [126]. The faint and fast novae could be associated to hot and massive white dwarfs [56], as predicted by [152]. The nova eruption is dominated by the mass of the white dwarf [61], but the role of composition, temperature and accretion rate must be included [56]. On the other hand, the identity of the absolute magnitude achieved 15 days after the maximum by all novae [12], [13], [20], [35], [141] is supported by the observation of M87 novae [128]. 
A new distance estimation method has been presented by [81]. The authors have estimated the distance of 73 novae and set limits for 46 objects. The method is based on the location of the red clump giants in the infrared color-magnitude diagram and the combination of reddening-distance relations and independent reddening estimates. The method has been calibrated against the distance of novae with known expansion parallax [35].

Recently, the first data release of GAIA has made available new estimations for the parallax of 16 cataclysmic variables [100], among them the parallax of three old novae: V603 Aql, RR Pic, HR Del. The GAIA parallaxes of V603 Aql, RR Pic are in broad agreement with the parallax estimation with HST, while the GAIA estimations for RR Pic and HR Del are slightly larger than expansion parallax distances.

\section{X-ray observations}

The availability of X-ray and gamma ray instrument has allowed to investigate new features of novae, among them the occurrence of shocks. Novae are X-ray emitters, due to the presence of accreting white dwarfs. The first X-ray observations with EXOSAT [75] and ROSAT [58] showed flux variations during the nova outbursts and a spectrum hardness evolving towards a super-soft phase. The hard and soft components are associated to shocks within the ejected material and to the nuclear burning on the white dwarf surface. Swift has produced high cadence light curves of novae, starting a few days after the outburst, for a large number of novae in the Galaxy, M31 and the Magellanic Clouds [80]. A rich pattern of emission lines has been discovered thanks to the high spectral resolution gratings of XMM-Newton and Chandra instruments [77], [73]. An example of X-ray light curve of a nova is given by the 2010 outburst of U Sco [82] (Fig. 6).
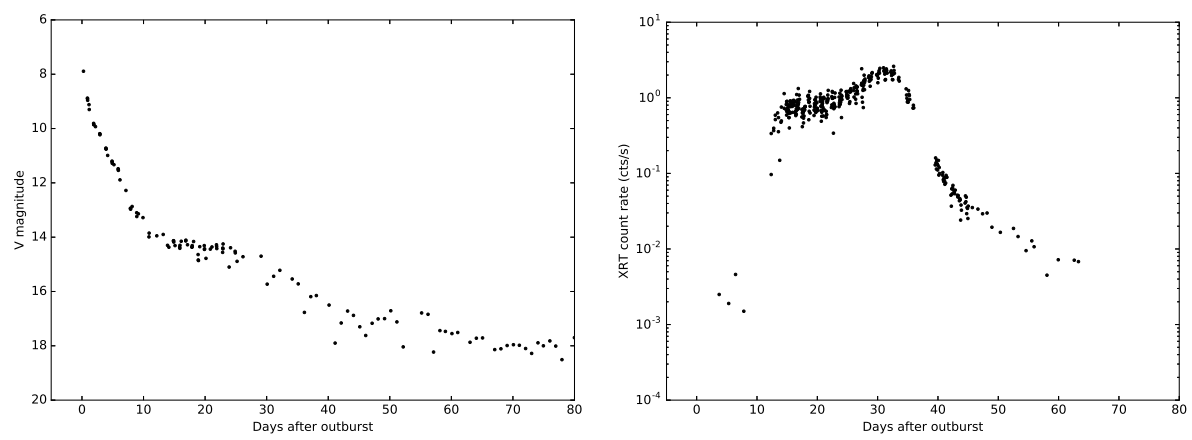

Figure 6: V band and Swift XRT light curve of U Sco during the 2010 eruption [82]; data from [82]

The monitoring of the 2006 outburst of RS Oph showed the presence of shocks of ejecta [8], [132], [79]. The radio interferometric [74] and HST observations [9] showed that the shock had a bipolar geometry with an equatorial ring. After the nova outburst, the keV X-ray flux initially faded before achieving a peak around day 30 , at the beginning of the super-soft phase. The shrinking of the photosphere and the decrease of the optical depth produced the exposure of the hotter layers, with the burning ending around day 80 and causing the decreasing of the X-ray flux.

Several novae detected by Swift have showed the super-soft phase [110]. An early turn-on and turn-off of the super-soft phase are associated to an high ejection velocity, thus to novae with a fast 
optical decline. The same relation has been found for novae in M31 [47]. The super-soft stage is often associated with the forbidden [Fe X] optical line at $6374 \AA$ [110]. The high resolution X-ray spectra are dominated by the continuum and the white dwarf absorption features or by strong and broad emission lines or both of them [77]. The thermal bremsstrahlung continuum observed by the first instruments shows emission lines ih the present high resolution instrumentation [76].

The spectra outside the super-soft phase are modeled with collisional plasmas [73]. The emission component before the beginning of the super-soft stage are explained by shocks, while the emission after the stage is caused by the radiatively cooling thin ejected material [73]. The universal decline law proposed by [44], [57] predicts the on and off times of the super-soft phase [45].

\section{Gamma ray observations}

Gamma ray emission in novae had been predicted at $\mathrm{MeV}$ energies, associated to positron annihilation and nuclear de-excitation in the decays of nitrogen, oxygen and sodium [19], [49]. The first detection of gamma rays in a nova unexpectedly occurred at high energy, when the FermiLAT instrument [7] observed the radiation of the symbiotic nova V407 Cyg in the region 0.1 to 10 $\mathrm{GeV}$ [1]. The energetic emission was explained by the interaction of material in the nova shell with the environmental medium of the red giant secondary. V407 Cyg is a symbiotic nova, where ejecta expand in a dense circumstellar wind and particles can be accelerated in a blast wave. After V407 Cyg, the Fermi-LAT detected GeV gamma ray emission in the classical novae V959 Mon, V1324 Sco and V339 Del [2]. The GeV light curve of V959 Mon is shown in Fig. 7.

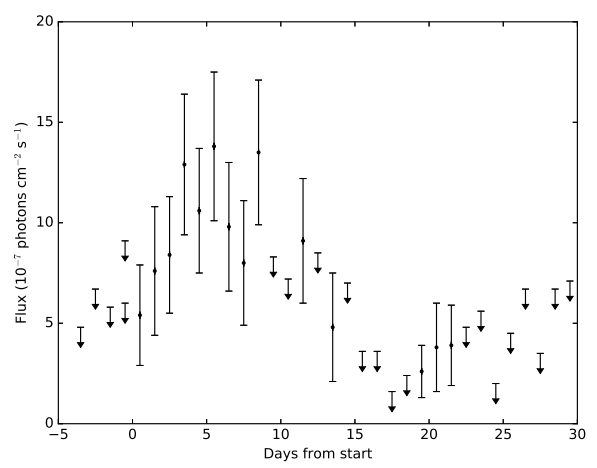

Figure 7: Fermi-LAT light curve (>100 MeV) [2]; data from [2]

The density of the environment around classical novae is smaller than around symbiotics: the acceleration is caused by a bow shock driven by the expelled material in the ISM or several small shocks due to turbulence [2]. V959 Mon, V1324 Sco and V339 Del all showed a soft spectrum over a few weeks. The observation of $\mathrm{GeV}$ gamma ray emission in different type of systems suggests that all novae could potentially emit gamma rays. The main models for gamma ray emission in novae are the hadronic and the leptonic scenarios [2]. In the former scenario, nova ejecta interact with nuclei in the environmental medium (novae) or with the stellar wind (symbiotics), producing neutral pions that decay to photons. In the latter scenario, gamma rays are produced by the in- 
teraction of accelerated electrons with photons through inverse Compton scattering or with atoms through bremsstrahlung. The Fermi-LAT instrument has observed $\mathrm{GeV}$ emission also from two recent novae, V1369 Cen and V5668 Sgr [16], detecting gamma rays from two days to some weeks after the outburst. The gamma ray emission lasted for a longer time, but was fainter, compared to V959 Mon, V1324 Sco and V339 Del [2]. Excluding the symbiotic nova V407 Cyg, the FermiLAT instrument has detected gamma rays from six novae, out of the 69 novae discovered in the optical domain during the same interval. According to [67], novae with a magnitude $\mathrm{R} \leq 12$ and within $8 \mathrm{kpc}$ are potentially detectable by the Fermi-LAT instrument.

Novae emitting gamma rays are suitable systems to investigate the physical mechanisms related to relativistic particle acceleration at non relativistic shocks [64]. The high density of ejected material at the epoch of gamma ray emission produces radiative shocks, the X-rays following the shock are absorbed in neutral gas and reprocessed into optical radiation. The fraction of the shock power spent in the acceleration of relativistic particles is related to the relative ratio of gamma ray and optical luminosity, For the novae V1324 Sco and V339 Del the ratio is larger than the value estimated from galactic cosmic rays. A prediction of the model is the emission of X-ray radiation in the range 10 to $100 \mathrm{keV}$, potentially detectable with NuSTAR, in coincidence with $\mathrm{GeV}$ emission [64]. The model for hard X-ray and gamma ray emission by [143] suggests that only a small fraction of the gamma ray luminosity is radiated in the NuSTAR energy band, but it could be detected in long exposures simultaneously with the GeV emission, showing the clear signature of a zero spectral slope in the $\mathrm{X}$-rays. The $\mathrm{GeV}$ emission of novae and the relation to particle acceleration at shocks has been addressed by [62], who suggested that it is mainly produced from particles accelerated up to $100 \mathrm{GeV}$ at the reverse shock and involved in hadronic interactions in the cooling layer downstream of the shock. The observation of gamma rays from nova ASASSN-16ma showed a correlation of gamma and optical radiation, suggesting that the former was mainly coming from the reprocessed emission from shocks [59]. The ratio of gamma to optical flux in ASASSN-16ma constrained the acceleration efficiency of non-thermal particles to as 0.005 , supporting hadronic models for the gamma ray emission. The shocks and the relativistic particle acceleration in the nova outburst [65] can produce gamma ray emission in the $\mathrm{TeV}$ region. The gas upstream the shock is neutral, since it is shielded by ionization and the acceleration zone is confined to a thin photo-ionized layer ahead the shock. The acceleration process predicts a maximum gamma ray energy in the range from $10 \mathrm{GeV}$ to $100 \mathrm{TeV}$ [65], the region of ground based atmospheric Cherenkov detectors, such as MAGIC or the future Cherenkov Telescope Array CTA [3]. The TeV gamma rays could be associated with $\mathrm{TeV}$ neutrinos, that are suitable sources for IceCube, as discussed by [131]. The Cherenkov instrument MAGIC has monitored several cataclysmic variables in the energy region above $50 \mathrm{GeV}$ [4]. No significant $\mathrm{TeV}$ emission was observed in V339 Del after its outburst. The combination of MAGIC and Fermi data of V339 Del showed that the total power related to accelerated protons was smaller than $15 \%$ of the total power related to accelerated electrons.

The optical light curve of seven novae emitting gamma rays is related to the gamma ray activity [72], showing two components. The first one is associated to the fireball stage, while the second component appears simultaneously with gamma rays and can provide an additional tool to investigate the high frequency emission of novae. 


\section{Conclusions}

Novae are multifrequency laboratories that involve a great variety of processes. Recently, observation of novae at high energy are providing new insights into the outburst and the successive evolution.

\section{Acknowledgements}

The author thanks the organizers for the invitation to the conference. Thanks to Ivan Bruni for the assistance and to the Time Allocation Committee of the Loiano telescope for the observing time.

\section{References}

[1] A. A. Abdo et al., Sci 329 (2010) 817.

[2] M. Ackermann et al., Sci 345 (2014) 554.

[3] M. Actis et al., ExA 32 (2001) 143.

[4] M. L. Ahnen et al., A\&A 582 (2015) 582.

[5] C. W. Allen, MNRAS 114 (1954) 387.

[6] H. C. Arp et al., AJ 61 (1956) 15.

[7] W. B. Atwood et al., ApJ 697 (2009) 1071.

[8] M. F. Bode et al., ApJ 652 (2006) 629.

[9] M. F. Bode at al., ApJ 665 (2007) L63.

[10] M. F. Bode and A. Evans, Classical Novae, Cambridge University Press (2012).

[11] D. Boyd et al., ATel 11116 (2017).

[12] W. Buscombe and G. de Vaucouleurs, Obs 75 (1955) 170.

[13] M. Capaccioli et al., AJ 97 (1989) 1622.

[14] M. Capaccioli et al., ApJ 360 (1990) 63.

[15] A. Cassatella and R. Viotti, Physics of Classical Novae, Springer-Verlag, Lecture Notes in Physics 369 (1990).

[16] C. C. Cheung et al., ApJ 826 (2016) 142.

[17] R. Ciardullo et al., ApJ 318 (1987) 520.

[18] R. Ciardullo et al., ApJ 356 (1990) 472.

[19] D. D. Clayton and F. Hoyle, ApJ 187 (1974) 101.

[20] J. G. Cohen, ApJ 292 (1985) 90.

[21] J. G. Cohen, in: The extragalactic distance scale, ASPC 4 (1988) 114.

[22] A. C. Collazzi et al., AJ 138 (2009) 1846.

[23] C. Curtin et al., ApJ 811 (2015) 34. 
[24] M. J. Darnley et al., MNRAS 369 (2006) 257.

[25] M. J. Darnley et al., ApJ 833 (2016) 149.

[26] G. de Vaucouleurs, ApJ 223 (1978) 351.

[27] M. Della Valle, in: The extragalactic distance scale, ASPC 4 (1988) 73.

[28] M. Della Valle et el., A\&A 266 (1992) 232.

[29] M. Della Valle et al., A\&A 287 (1994) 403.

[30] M. Della Valle, in Classical Nova Explosions, AIPC 637 (2002) 443.

[31] M. Della Valle and R. Gilmozzi, Sci 296 (2002) 1275.

[32] M. della Valle and M. Livio, A\&A 286 (1994) 786.

[33] M. della Valle and M. Livio, ApJ 452 (1995) 704.

[34] M. della Valle and M. Livio, ApJ 506 (1998) 818.

[35] R. A. Downes and H. W. Duerbeck, AJ 120 (2000), 2007.

[36] R. A. Downes et al., JAD 11 (2005) 2

[37] H. W. Duerbeck, SSR 45 (1987) 1.

[38] H. W. Duerbeck, MNRAS 258 (1992) 629.

[39] H. W. Duerbeck, AN 330 (2009) 568.

[40] A. Ederoclite, in: Stella Novae: Past and Future Decades, ASPC 490 (2014) 163.

[41] F. Giovannelli and L. Sabau-Graziati, in Proceedings of The Golden Age of Cataclysmic Variables and Related Objects - III, POS (Golden2015) 001 (2016).

[42] J. A. Graham, in Changing Trends in Variable Star Research, IAU Coll. 46 (1979) 96.

[43] M. A. Guerrero et al., ApJ 857 (2018) 80.

[44] I. Hachisu and M. Kato, ApJSS 167 (2006) 59.

[45] I. Hachisu and M. Kato, ApJ 709 (2010) 680.

[46] K. Hatano et al., MNRAS 290 (1997) 113.

[47] M. Henze et al., A\&A 563 (2014) A2.

[48] N. Henze et al., $A \& A \mathbf{5 8 2}$ (2015) 8.

[49] M. Hernanz, in Stella Novae: Past and Future Decades, ASPC 490 (2014) 319.

[50] R. Hounsell et al., ApJ 734 (2010) 480.

[51] R. Hounsell et al., ApJ 820 (2016) 104.

[52] E. P. Hubble, ApJ 69 (1929) 103.

[53] R. Hudec, in Proceedings of The Golden Age of Cataclysmic Variables and Related Objects - III, PoS (Golden2015) 041 (2016).

[54] K. Imamura and K. Tanabe, PASJ 64 (2012) L9.

[55] K. Itagaki et al., ATel 9848 (2016). 
[56] M. M. Kasliwal et al., ApJ 735 (2011) 94.

[57] M. Kato and I. Hachisu, ApJ 437 (1994) 803.

[58] J. Krautter et al., ApJ 456 (1996) 788.

[59] K.-L. Li et al., NatAs 1 (2017) 697.

[60] W. Liller and B. Mayer, PASP 99 (1987) 600.

[61] M. Livio, ApJ 393 (1992) 516.

[62] P. Martin et al., $A \& A 612$ (2018) A38.

[63] F. Matteucci et al., $A \& A 405$ (2003) 23.

[64] B. D. Metzger et al., MNRAS 450 (2015) 2739.

[65] B. D. Metzger et al., MNRAS 457 (2016) 1786.

[66] B. Miszalski et al., MNRAS 456 (2016) 633.

[67] P. J. Morris et al., MNRAS 495 (2017) 1218.

[68] P. Mroz et al., ApJS 219 (2015) 26.

[69] P. Mroz et al., ApJS 232 (2016) 9.

[70] P. Mroz et al., Nat 537 (2016) 649.

[71] https://asd.gsfc.nasa.gov/Koji.Mukai/novae/novae.html

[72] U. Munari et al., MNRAS 469 (2017) 4341.

[73] J. U. Ness, BASI 40 (2012) 353.

[74] T. J. O'Brien et al., Nat 442 (2006) 279.

[75] H. Ögelman et al., A\&A 177 (1987) 110.

[76] M. Orio et al., ApJ 466 (1995) 410.

[77] M. Orio, BASI 40 (2012) 333.

[78] M. Orio, in Proceedings of The Golden Age of Cataclysmic Variables and Related Objects - III, PoS (Golden2015) 064 (2016).

[79] J. P. Osborne eta al., ApJ 727 (2011) 124.

[80] J. P. Osborne, JHEA 7 (2015) 117.

[81] A. Özdönmez et al., MNRAS 461 (2016) 1177.

[82] A. Pagnotta et al., AJ 811 (2015) 32.

[83] A. Pagnotta, in 20th European White Dwarf Workshop, ASPC 509 (2017) 535.

[84] J. Patterson, SASS 33 (2014) 17.

[85] C. H. Payne-Gaposchkin, The Galactic Novae, North-Holland Pub. Co. (1957).

[86] W. Pietsch, AN 331 (2010) 187.

[87] R. Poggiani, AN 327 (2006) 895.

[88] R. Poggiani, NewA 13 (2008) 557. 
[89] R. Poggiani, Ap\&SS 315 (2008) 79.

[90] R. Poggiani, NewA 14 (2009) 4.

[91] R. Poggiani, AN 330 (2009) 77.

[92] R. Poggiani, Ap\&SS 323 (2009) 319.

[93] R. Poggiani, NewA 15 (2010) 657.

[94] R. Poggiani, NewA 15 (2010) 170.

[95] R. Poggiani, Ap\&SS 333 (2011) 115.

[96] R. Poggiani, MmSAI 83 (2012) 753.

[97] R. Poggiani, NewA 37 (2015) 9.

[98] R. Poggiani, AcPPP 2 (2015) 234.

[99] R. Poggiani,in: Proceedings of The Golden Age of Cataclysmic Variables and Related Objects IV (GOLDEN 2017), POS (GOLDEN 2017) 049 (2018).

[100] G. Ramsay et al., $A \& A 604$ (2017) A107.

[101] H. Ritter and U. Kolb, A\&A 404 (2003) 301.

[102] http://wwwmpa.mpa-garching.mpg.de/RKcat/

[103] E. L. Robinson, $A J 80$ (1975) 515.

[104] L. Rosino, AnAp 27 (1964) 498.

[105] L. Rosino, A\&AS 9 (1973) 347.

[106] L. Rosino, AJ 97 (1989) 83.

[107] D. I. Sahman et al., MNRAS 477 (2018) 4483.

[108] B. E. Schaefer, ApJS 187 (2010) 275.

[109] T. Schmidt, Z. Astrophys. 41 (1957) 182.

[110] G. Schwarz et al., ApJSS 197 (2011) 31.

[111] A. W. Shafter, ApJ 487 (1997) 226.

[112] A. W. Shafter et al., ApJ 530 (2000) 193S.

[113] A. W. Shafter, in: Classical Nova Explosions: International Conference on Classical Nova Explosions, AIPC 637 (2002) 462.

[114] A. W. Shafter et al., ApJ 734 (2011) 12.

[115] A. W. Shafter et al., ApJ 752 (2012) 156.

[116] A. W. Shafter, $A J 145$ (2013) 117.

[117] A. W. Shafter, in: Stella Novae: Past and Future Decades, ASPC 490 (2014) 77.

[118] A. W. Shafter et al., ApJS 216 (2015) 34.

[119] A. W. Shafter, ApJ 834 (2017) 196.

[120] A. W. Shafter and P. K. Irby, ApJ 563 (2001) 749. 
[121] M. M. Shara et al., ApJ 311 (1986) 163.

[122] M. M. Shara et al., Nat 446 (2007) 159.

[123] M.M. Shara et al.,ApJ 758 (2012) 121.

[124] M. M. Shara et al., ApJS 227 (2016) 1.

[125] M. M. Shara et al., Nat 548 (2017) 558.

[126] M. M. Shara et al., ApJ 839 (2017) 109.

[127] M. M. Shara et al., MNRAS 465 (2017) 739.

[128] M. M. Shara et al., MNRAS 474 (2018) 1746.

[129] A. S. Sharov, SvA 16 (1972) 41.

[130] A. S. Sharov, AstL 19 (1993) 147.

[131] J. Sitarek and W. Bednarek, PRD 86 (2012) 063011.

[132] J. J. Sokoloski et al., Nat 442 (2006) 276.

[133] S. Starrfield et al., PASP 128 (2016) 051001.

[134] R. J. Strope et al., AJ 140 (2010) 34.

[135] F. Surina et al., arXiv:1111.5524 (2011).

[136] J. Tanaka et al., PASJ 63 (2011) 911.

[137] C. Tappert et al., MNRAS 423 (2012) 2476.

[138] C. Tappert et al., in: Proceedings of The Golden Age of Cataclysmic Variables and Related Objects III, PoS (Golden2015) 062 (2016).

[139] A. B. Tomaney and A. W. Shafter, ApJS 81 (1992) 683.

[140] S. van den Bergh and C. J. Pritchet, PASP 98 (1986) 110.

[141] S. van den Bergh and P. F. Younger, $A \& A S 70$ (1987) 125.

[142] S. van den Bergh, PASP 103 (1991) 609.

[143] I. Vurm and B. D. Metzger, ApJ 852 (2018) 62.

[144] F. M. Walter et al., PASP 124 (2012) 1057.

[145] F. M. Walter, in Stella Novae: Past and Future Decades, ASPC 490 (2014) 191.

[146] R. E. Williams et al., ApJ 376 (1991) 721.

[147] R. E. Williams, AJ 104 (1992) 725.

[148] R. E. Williams et al., ApJSS 90 (1994) 297.

[149] R. E. Williams, JAD 9 (2003).

[150] R. E. Williams and A. W. Shafter, ApJ 612 (2004) 867.

[151] R. Williams, AJ 144 (2012) 98.

[152] O. Yaron et al., ApJ 623 (2005) 398.

[153] L. Yungelson et al., ApJ 481 (1997) 127. 